\title{
Photon-pion transition form factor within nonlocal chiral quark model
}

\author{
A. Dorokhov* \\ Bogoliubov Laboratory of Theoretical Physics, Russia \\ E-mail: dorokhov@theor.jinr.ru
}

\begin{abstract}
Recently, the BABAR collaboration reported the measurments of the photon-pion transition form factor $F_{\pi \gamma \gamma^{*}}\left(Q^{2}\right)$, which are in strong contradiction to the predictions of the standard factorization approach to perturbative QCD. In the present talk, based on a nonperturbative approach to the QCD vacuum and on rather universal assumptions, we show that there exists two asymptotic regimes for the pion transition form factor. One regime with asymptotics $F_{\pi \gamma^{*} \gamma}\left(Q^{2}\right) \sim 1 / Q^{2}$ corresponds to the result of the standard QCD factorization approach, while other violates the standard factorization and leads to asymptotic behavior as $F_{\pi \gamma^{*} \gamma}\left(Q^{2}\right) \sim \ln \left(Q^{2}\right) / Q^{2}$. Furthermore, considering specific nonlocal chiral quark models, we find the region of parameters, where the existing CELLO, CLEO and BABAR data for the pion transition form factor are successfully described.
\end{abstract}

Light Cone 2010: Relativistic Hadronic and Particle Physics

June 14-18, 2010

Valencia, Spain

${ }^{*}$ Speaker. 


\section{Introduction}

The theory of hard exclusive processes, formulated within the factorization approach to perturbative quantum chromodynamics (pQCD), is based on the operator product expansion (OPE), the factorization theorems, and the pQCD evolution equations. In this context, the form factor for the photon-pion transition $\gamma^{*} \gamma^{*} \rightarrow \pi^{0}$, with both photons being spacelike (with photon virtualities $Q_{1}^{2}, Q_{2}^{2}>0$ ), was considered in [1,2]. Since only one hadron is involved, the corresponding form factor $F_{\pi \gamma^{*} \gamma^{*}}\left(Q_{1}^{2}, Q_{2}^{2}\right)$ has the simplest structure for the pQCD analysis among the hard exclusive processes. The nonperturbative information about the pion is accumulated in the pion distribution amplitude (DA) $\varphi_{\pi}(x)$ for the fraction $x$ of the longitudinal pion momenta $p$, carried by a quark. Another simplification is, that the short-distance amplitude for the $\gamma^{*} \gamma^{*} \rightarrow \pi^{0}$ transition is, to leading order, just given by a single quark propagator. Finally, the photon-pion form factor is related to the axial anomaly, when both photons are real.

Experimentally, the easiest situation is, when one photon virtuality is small and the other large. Under these conditions, the form factor $F_{\pi \gamma^{*} \gamma}\left(Q^{2}, 0\right)$ was measured at $e^{+} e^{-}$colliders by CELLO [3], CLEO [4] Collaborations (Fig. 1). In the region of large virtualities $Q^{2}>>1 \mathrm{GeV}^{2}$, the pQCD factorization approach for exclusive processes predicts to leading order in the strong coupling constant $[1,2]$

$$
F_{\pi \gamma^{*} \gamma}^{\mathrm{pQCD}}\left(Q^{2}, 0\right)=\frac{2 f_{\pi}}{3 Q^{2}} J
$$

where $J=\int_{0}^{1} d x x^{-1} \varphi_{\pi}(x)$ is the inverse moment of the pion DA, and $f_{\pi}=92.4 \mathrm{MeV}$. The factor $1 / Q^{2}$ reflects the asymptotic property of the quark propagator connecting two quark-photon vertices (Fig. 2). The formula (1.1) is derived under the assumption, that the QCD dynamics at large distances (the factor $J f_{\pi}$ ) and the QCD dynamics at small distances (the factor $1 / Q^{2}$ ) is factorized. Moreover, under this assumption, the asymptotics is reached already at the typical hadronic scale of a few $\mathrm{GeV}^{2}$. The pion DA $\varphi_{\pi}(x)$, in addition, evolves in shape with the change of the renormalization scale and asymptotically equals $\varphi_{\pi}^{\mathrm{As}}(x)=6 x(1-x)$. From this follows the famous asymptotic prediction (the straight dotted line in Fig. 1)

$$
F_{\pi \gamma^{\gamma} \gamma}^{\mathrm{pQCD}, \mathrm{As}}\left(Q^{2}, 0\right)=\frac{2 f_{\pi}}{Q^{2}}
$$

Recently, the BABAR collaboration published new data (Fig. 1) for the $\gamma \gamma^{*} \rightarrow \pi^{0}$ transition form factor in the momentum transfer range from 4 to $40 \mathrm{GeV}^{2}$ [5]. They found the following puzzling result: At $Q^{2}>10 \mathrm{GeV}^{2}$ the measured form factor multiplied by the photon virtuality $Q^{2} F_{\pi \gamma^{*} \gamma}\left(Q^{2}, 0\right)$ exceeds the predicted asymptotic limit (1.2) and, moreover, continues to grow with increasing $Q^{2}$. This result is in strong contradiction to the predictions of the standard QCD factorization approach mentioned above. The BABAR data very well match the older data obtained by the CLEO collaboration in the smaller $Q^{2}$ region, but extend to a much lager $Q^{2}$ values.

\section{Nonlocal chiral quark model}

We will analyze the photon-pion transition form factor in the gauged nonlocal chiral quark model based on the picture of nontrivial QCD vacuum. The attractive feature of this model is, 

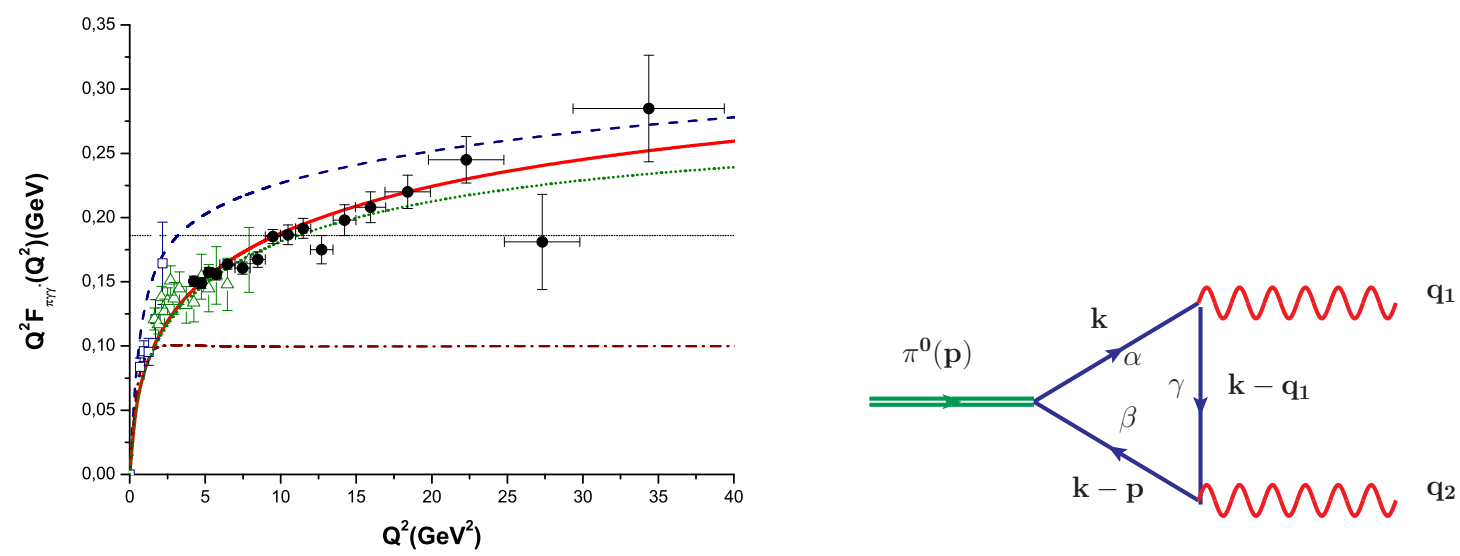

Figure 2: The triangle diagram in momentum

Figure 1: Photon-pion transition form factor in asymmetric kinemat- and $\alpha$-representation notation. ics for the instanton model with parameters $M_{q}=125 \mathrm{MeV}, \Lambda=0.016$ $\mathrm{GeV}^{-2}$ (short pointed line), $M_{q}=300 \mathrm{MeV}, \Lambda=1.3 \mathrm{GeV}^{-2}$ (dashdotted line); and chiral model with parameters $M_{q}=125 \mathrm{MeV}, \Lambda=$ $0.0098 \mathrm{GeV}^{-2}$ (solid line) and $M_{q}=300 \mathrm{MeV}, \Lambda=0.639 \mathrm{GeV}^{-2}$ (dashed line). The straight dotted line is asymptotic limit $2 f_{\pi}$. The data points are from the CELLO [3] (empty squares), CLEO [4] (empty triangles) and BABAR (filled circles) [5] Collaborations.

that it interpolates the physics at large and small distances. At low energy, it enjoys the spontaneous breaking of chiral symmetry, the generation of the dynamical quark mass, and it satisfies the basic low energy theorems. In particular, the correct normalization of the form factor by the axial anomaly $F_{\pi \gamma \gamma}(0,0)=1 /\left(4 \pi^{2} f_{\pi}\right)$, and the Goldberger-Treiman relation, connecting the quark-pion coupling $g_{q \pi}$ and the dynamical quark mass $M_{q}$ with the physical pion decay constant $f_{\pi}: f_{\pi}=M_{q} / g_{q \pi}$. At energies much higher than the characteristic hadronic scale, it becomes the theory of free massless quarks (in chiral limit).

Let us discuss the properties of the triangle diagram (Fig. 2) at large photon virtualities. To this end, we do not need to completely specify the elements of the diagram technique, which are, in general, model dependent, but shall restrict ourselves to rather general requirements. All expressions will be treated in Euclidean space appropriate for the process under consideration and for the treatment of nonperturbative physics. The nonperturbative quark propagator, dressed by the interaction with the $Q C D$ vacuum, is

$$
S(k)=\frac{\widehat{k}+m\left(k^{2}\right)}{D\left(k^{2}\right)} .
$$

The main requirement to the quark propagator is, that at large quark virtualities $k^{2} \rightarrow \infty$ one has

$$
S(k) \rightarrow \frac{\widehat{k}}{k^{2}} \text {. }
$$

We assume also, that the dynamical quark mass is a function of the quark virtuality $k^{2}$ and normalized at zero as $m(0)=M_{q}$. At large virtualities, it drops to the current quark mass $m_{\text {curr }}$ faster than 
any power of $k^{-2}$ (see the discussion in [6])

$$
m\left(k^{2}\right) \sim M_{q} \exp \left(-\left(k^{2}\right)^{a}\right)+m_{\text {curr }}, \quad a>0 .
$$

The denominator in (2.1) at large virtualities $k^{2} \rightarrow \infty$ is $D\left(k^{2}\right) \rightarrow k^{2}$.

It is well known (see, e.g., $[7,8]$ ), that the change of the quark propagator leads to a modification of the quark-photon vertex in order to preserve the Ward-Takahashi identity

$$
\Gamma_{\mu}\left(k, q, k^{\prime}=k+q\right)=-i e_{q}\left[\gamma^{\mu}-\Delta \Gamma_{\mu}\left(k, q, k^{\prime}=k+q\right)\right] .
$$

The term $\Delta \Gamma_{\mu}(q)$ is not uniquely defined, even within a particular model, especially its transverse part. The importance of the full vertex $\Gamma_{\mu}$ is, that the axial anomaly is reproduced [9], and thus the photon-transition form factor correctly normalized. Fortunately, due to the fact, that $\Delta \Gamma_{\mu}$ is not proportional to $\gamma_{\mu}$ matrix, the corresponding amplitude has no projection onto the leading twist operator. Thus, this term is suppressed, if a large photon virtuality passes through the vertex, and hence does not participate in the leading asymptotics of the form factor. Its leading asymptotics results exclusively from the local part of the photon vertex

$$
\Gamma_{\mu}^{\mathrm{As}}\left(k, q, k^{\prime}=k+q\right)=-i e_{q} \gamma^{\mu}
$$

Furthermore, we need the quark-pion vertex,

$$
\Gamma_{\pi}^{a}(p)=\frac{i}{f_{\pi}} \gamma_{5} \tau^{a} F\left(k_{+}^{2}, k_{-}^{2}\right)
$$

where $k_{+}$and $k_{-}$are the quark and antiquark momenta. In the following, the important feature of the vertex function $F\left(k_{+}^{2}, k_{-}^{2}\right)$ will be its behavior in the limit, when one quark virtuality is asymptotically large (e.g., $k_{-}^{2} \rightarrow \infty$ ) and the other $\left(k_{+}^{2}\right)$ remains finite. There are two possibilities,

$$
F^{f}\left(k_{+}^{2}, k_{-}^{2}\right) \rightarrow 0
$$

and

$$
F^{u f}\left(k_{+}^{2}, k_{-}^{2}\right) \rightarrow g\left(k_{+}^{2}\right) .
$$

Finally, one needs the projection of the pion state onto the leading twist operator

$$
\Gamma_{\mu}^{5, \mathrm{As}}\left(k, q, k^{\prime}=k+q\right)=\gamma^{\mu} \gamma^{5}
$$

This projection is determined by the matrix element $\left\langle 0\left|\bar{q} \gamma^{\mu} \gamma^{5} \tau^{a} q\right| \pi^{a}(p)\right\rangle=-i 2 f_{\pi, \mathrm{PS}}$, where the constant $f_{\pi, \mathrm{PS}}$ is (here $m^{\prime}(u)=d m(u) / d u$ )

$$
f_{\pi, \mathrm{PS}}^{2}=\frac{N_{c}}{4 \pi^{2}} \int_{0}^{\infty} d u \quad u \frac{F(u, u)}{D^{2}(u)}\left(m(u)-\frac{1}{2} u m^{\prime}(u)\right)
$$

which coincides with the pion decay constant $f_{\pi, \mathrm{PS}}$ in the Pagels-Stokar form [11]. 


\section{Asymptotics of pion-photon transition form factor}

The invariant amplitude for the process $\gamma^{*} \gamma^{*} \rightarrow \pi^{0}$ is given by

$$
A\left(\gamma^{*}\left(q_{1}, \varepsilon_{1}\right) \gamma^{*}\left(q_{2}, \varepsilon_{2}\right) \rightarrow \pi^{0}(p)\right)=-i e^{2} \varepsilon_{\mu v \rho \sigma} \varepsilon_{1}^{\mu} \varepsilon_{2}^{v} q_{1}^{\rho} q_{2}^{\sigma} F_{\pi \gamma^{*} \gamma^{*}}\left(-q_{1}^{2},-q_{2}^{2}\right),
$$

where $\varepsilon_{i}^{\mu}$ are the photon polarization vectors, $p^{2}=m_{\pi}^{2}, q_{1}^{2}=-Q_{1}^{2}, q_{2}^{2}=-Q_{2}^{2}$. In the effective nonlocal quark-model considered above, one finds the contribution of the triangle diagram to the invariant amplitude [10],

$$
A\left(p^{2} ; q_{1}^{2}, q_{2}^{2}\right)=A^{\text {loc }}\left(p^{2} ; q_{1}^{2}, q_{2}^{2}\right)+A^{\text {nonloc }}\left(p^{2} ; q_{1}^{2}, q_{2}^{2}\right),
$$

where the first term contains only local part of the photon vertices and the second term comprises the rest.

As we discussed above, the leading asymptotics results from the local part of the amplitude, $A^{\text {loc }}$. After taking the Dirac trace one obtains

$$
A^{\mathrm{loc}}\left(p^{2} ; q_{1}^{2}, q_{2}^{2}\right)=\frac{e^{2} N_{c}}{6 \pi^{2} f_{\pi}} \int \frac{d^{4} k}{\pi^{2}} F\left(k_{+}^{2}, k_{-}^{2}\right) \frac{m\left(k_{+}^{2}\right)\left(\varepsilon_{12 k q_{2}}-\varepsilon_{12 q_{1} q_{2}}\right)-m\left(k_{-}^{2}\right) \varepsilon_{12 q_{1} k}+m\left(k_{3}^{2}\right) \varepsilon_{12 p k}}{D\left(k_{+}^{2}\right) D\left(k_{-}^{2}\right) D\left(k_{3}^{2}\right)},
$$

where $p=q_{1}+q_{2}, q=q_{1}-q_{2}, k_{ \pm}=k \pm p / 2, k_{3}=\left(k_{+}-q_{1}\right)$, and $\varepsilon_{12 k q_{2}}=\varepsilon_{\mu \nu \lambda \rho} \varepsilon_{1}^{\mu} \varepsilon_{2}^{v} k^{\lambda} q_{2}^{\rho}$, etc.

In order to analyze the asymptotic properties of the form factor, let us transform the integral in (3.2) formally into the $\alpha$ representation. Let us define for any function $F$ of virtuality $k^{2}$, decaying at large virtuality as $1 / k^{2}$ or faster, its $\alpha$ representation (Laplace transform)

$$
F\left(k^{2}\right)=\int_{0}^{\infty} d \alpha e^{-\alpha k^{2}} f(\alpha), \quad F\left(k^{2}\right) \sim f(\alpha),
$$

where $F\left(k^{2}\right)$ is the image of the original $f(\alpha)$. Then, the momentum integral in (3.2) is transformed into the following expression for the form factor (in the chiral limit)

$$
\begin{aligned}
F_{\pi \gamma^{*} \gamma^{*}}^{\mathrm{loc}}\left(p^{2}=0 ; Q_{1}^{2}, Q_{2}^{2}\right) & =\frac{N_{c}}{6 \pi^{2} f_{\pi}} \int \frac{d(\alpha \beta \gamma)}{\Delta^{3}} e^{-\frac{1}{\Delta} \gamma\left(\alpha Q_{1}^{2}+\beta Q_{2}^{2}\right)} \\
& \cdot\left[d(\gamma)\left(\alpha G_{m, 0}(\alpha, \beta)+\beta G_{0, m}(\alpha, \beta)\right)+\gamma d_{m}(\gamma) G(\alpha, \beta)\right],
\end{aligned}
$$

where $\Delta=\alpha+\beta+\gamma$ and $\int d(\alpha \beta \gamma) \ldots=\int_{0}^{\infty} d \alpha \int_{0}^{\infty} d \beta \int_{0}^{\infty} d \gamma \ldots$ In (3.4) we introduce the following notations

$$
\begin{aligned}
& \frac{1}{D\left(k^{2}\right)} \sim d(\alpha), \quad \frac{m\left(k^{2}\right)}{D\left(k^{2}\right)} \sim d_{m}(\alpha), \\
& \frac{F\left(k_{+}^{2}, k_{-}^{2}\right)}{D\left(k_{+}^{2}\right) D\left(k_{-}^{2}\right)} \sim G(\alpha, \beta), \quad \frac{m\left(k_{+}^{2}\right) F\left(k_{+}^{2}, k_{-}^{2}\right)}{D\left(k_{+}^{2}\right) D\left(k_{-}^{2}\right)} \sim G_{m, 0}(\alpha, \beta) .
\end{aligned}
$$

\subsection{Asymmetric kinematics I}

Let us now consider the asymmetric kinematics $Q_{1}^{2}=Q^{2}, Q_{2}^{2}=0$. Then one has

$$
\begin{aligned}
F_{\pi \gamma^{*} \gamma}^{\text {loc }}\left(0 ; Q^{2}, 0\right) & =\frac{N_{c}}{6 \pi^{2} f_{\pi}} \int \frac{d(\alpha \beta \gamma)}{\Delta^{3}} e^{-\frac{\gamma \alpha}{\Delta} Q^{2}} \\
& \cdot\left[d(\gamma)\left(\alpha G_{m, 0}(\alpha, \beta)+\beta G_{0, m}(\alpha, \beta)\right)+\gamma d_{m}(\gamma) G(\alpha, \beta)\right] .
\end{aligned}
$$


Let us first consider the model with the quark-pion vertex possessing the property (2.7). The leading large $Q^{2}$ behavior corresponds to the integral over small $\gamma$ and we get for $Q^{2} \rightarrow \infty$

$$
F_{\pi \gamma^{*} \gamma}^{\mathrm{loc} I}\left(0 ; Q^{2}, 0\right)=\frac{N_{c}}{6 \pi^{2} f_{\pi}} \int \frac{d(\alpha \beta)}{(\alpha+\beta)^{3}} \frac{\alpha G_{m, 0}(\alpha, \beta)+\beta G_{0, m}(\alpha, \beta)}{D\left(\frac{\alpha Q^{2}}{\alpha+\beta}\right)} .
$$

After change of variables $\alpha \rightarrow x L, \beta \rightarrow(1-x) L$, we arrive at the representation

$$
F_{\pi \gamma^{*} \gamma}^{\mathrm{loc}, \mathrm{I}}\left(0 ; Q^{2}, 0\right)=\frac{2}{3} \frac{f_{\mathrm{PS}, \pi}^{2}}{f_{\pi}} \int_{0}^{1} d x \frac{1}{D\left(x Q^{2}\right)} \varphi_{\pi}^{f}(x),
$$

where the pion distribution amplitude is

$$
\varphi_{\pi}(x)=\frac{N_{c}}{4 \pi^{2} f_{\mathrm{PS}, \pi}^{2}} \int_{0}^{\infty} \frac{d L}{L} e^{x \bar{x} L p^{2}}\left(x G_{m, 0}(x L, \bar{x} L)+\bar{x} G_{0, m}(x L, \bar{x} L)\right),
$$

Because in the considered case $\varphi_{\pi}(x)$ vanishes at the endpoints the actual asymptotics is in agreement with (1.1).

As we have already noted in Introduction the asymptotic behavior (1.1) is not seen in the BABAR data. Nevertheless, even for the case considered, in principle, it is possible to simulate in some wide preasymptotic kinematical region a logarithmically enhanced behavior of the form factor. This happens if one assumes that the pion DA entering (3.8) is almost flat $\varphi_{\pi}(x) \approx 1$, i.e. it is close to a constant everywhere except small vicinity near endpoints. Then, nonfactorizable asymptotic coefficient $J^{f}$ appears [12]

$$
J^{L}=Q^{2} \int_{0}^{1} d x \frac{1}{D\left(x Q^{2}\right)} .
$$

Let us consider some popular models of the nonperturbative quark propagator

$$
\begin{aligned}
& \frac{1}{D\left(k^{2}\right)}=\frac{1-\exp \left(-k^{2} / \Lambda^{2}\right)}{k^{2}} \\
& D\left(k^{2}\right)=k^{2}+m^{2}(k) .
\end{aligned}
$$

The first expression has the property of analytical confinement $[13,14]$ and the second one is typical for chiral models. In quark models, where the first propagator is used, the parameter $\Lambda$ has the meaning of a dynamical quark mass [15], $\Lambda \equiv M_{q}$, with typical values of $M_{q}=200-300 \mathrm{MeV}$. Inserting (3.11) into (3.10) it is possible to show that the leading asymptotic behavior as $Q^{2} \rightarrow \infty$

$$
J_{A C}^{L}=\ln \left(Q^{2} / M_{q}^{2}\right)+\text { const }
$$

This result (3.13) is very close to the result obtained in [16] (Gaussian and logarithmic models), where the idea of flat pion distribution amplitude for explanation of the BABAR data was suggested (see also $[17,18]$ ). 


\subsection{Asymmetric kinematics II}

Now, let us consider the model with the quark-pion vertex possessing the property (2.8). It is convenient to rearrange the terms in the pion form factor in the following way

$$
\begin{aligned}
& F_{\pi \gamma^{*} \gamma}^{\mathrm{loc}, \mathrm{II}}\left(0 ; Q^{2}, 0\right)=\frac{N_{c}}{6 \pi^{2} f_{\pi}} \int \frac{d(\alpha \beta \gamma)}{\Delta^{3}} e^{-\frac{\gamma \alpha}{\Delta} Q^{2}}\left\{\beta r_{m}(\beta)\right. \\
& +\alpha G_{m, 0}(\alpha, \beta) d(\gamma)+\beta\left[G_{0, m}(\alpha, \beta)-r_{m}(\beta)\right] \\
& +\gamma G(\alpha, \beta) d_{m}(\gamma)+\beta r_{m}(\beta)[d(\gamma)-1] \\
& \left.+\beta[d(\gamma)-1]\left[\beta G_{0, m}(\alpha, \beta)-r_{m}(\beta)\right]\right\}
\end{aligned}
$$

where we introduce notations for the originals

$$
\frac{g\left(k^{2}\right)}{D\left(k^{2}\right)} \sim r(\alpha), \quad \frac{m\left(k^{2}\right) g\left(k^{2}\right)}{D\left(k^{2}\right)} \sim r_{m}(\alpha) .
$$

After standard manipulations with the integrals one obtains the following large- $Q^{2}$ asymptotic behavior as $Q^{2} \rightarrow \infty$ transformed to the momentum representation [12]

$$
\begin{aligned}
& F_{\pi \gamma^{*} \gamma}^{A s, \mathrm{II}}\left(0 ; Q^{2}, 0\right)=\frac{1}{Q^{2}} \frac{N_{c}}{6 \pi^{2} f_{\pi}}\left[\int_{0}^{\infty} d u \frac{m(u) g(u)}{D(u)} \ln \left(\frac{Q^{2}}{u}\right)+A\right], \\
& A=\int_{0}^{\infty} d u \frac{1}{D(u)} \int_{0}^{1} d y \frac{m(y u)}{D(y u)}\left\{u F^{u f}(u, y u)-\left[u+2 m^{2}(u)\right] g(y u)\right\} .
\end{aligned}
$$

The asymptotic expression (3.15) generalizes the asymptotic formula (1.1) for the case when the standard factorization is violated.

\section{The instanton and chiral models}

In the previous section we considered the asymptotic behavior of the pion transition form factor. In order to calculate this form factor in the whole kinematic region and compare with available experimental data, we should further specify our model assumptions. Let us introduce the momentum-dependent dynamical quark mass entering the propagator (2.1) as

$$
m\left(k^{2}\right)=M_{q} f^{2}\left(k^{2}\right)
$$

and take the profile function $f\left(k^{2}\right)$ in a Gaussian form $f\left(k^{2}\right)=\exp \left(-\Lambda k^{2}\right)$. Thus, the model contains two parameters, the dynamical quark mass $M_{q}$ and the non-locality parameter $\Lambda$.

Next, we need to specify the nonlocal part of the vector vertex that does not participate in the leading asymptotics, but is very important in implementing the low energy theorems. The nonlocal part of the vector vertex in (2.4) is taken of the form [7]

$$
\Delta \Gamma_{\mu}\left(k, q, k^{\prime}=k+q\right)=\left(k+k^{\prime}\right)_{\mu} \frac{m\left(k^{\prime 2}\right)-m\left(k^{2}\right)}{k^{\prime 2}-k^{2}} .
$$

Further, we will consider two kinds of quark-pion vertex (2.6), the first given by

$$
F_{I}\left(k_{+}^{2}, k_{-}^{2}\right)=M_{q} f\left(k_{+}^{2}\right) f\left(k_{-}^{2}\right),
$$


and the second by

$$
F_{\chi}\left(k_{+}^{2}, k_{-}^{2}\right)=\frac{1}{2} M_{q}\left[f^{2}\left(k_{+}^{2}\right)+f^{2}\left(k_{-}^{2}\right)\right] .
$$

The first one is motivated by the instanton picture of QCD vacuum [19] and the second by the nonlocal chiral quark model advertised in [20]. We shall in the further discussion refer to vertex function (4.3), which has the $k^{2} \rightarrow \infty$ behavior (2.7), as the instanton model, and to the other choice (4.4), corresponding to $k^{2} \rightarrow \infty$ behavior(2.8), as the chiral model.
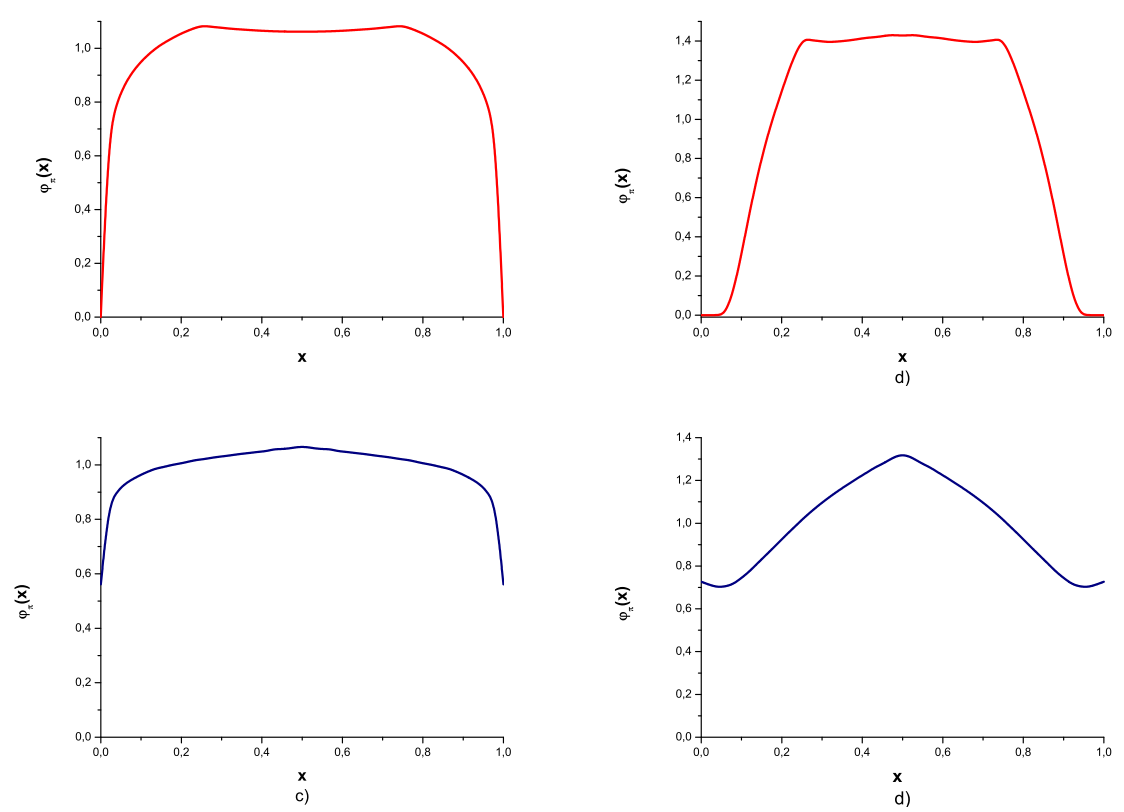

Figure 3: Pion distribution amplitude for the instanton model with parameters a) $M_{q}=125 \mathrm{MeV}, \Lambda=0.016 \mathrm{GeV}^{-2}$ and b) $M_{q}=300 \mathrm{MeV}, \Lambda=1.3 \mathrm{GeV}^{-2}$; and chiral model with parameters c) $M_{q}=125 \mathrm{MeV}, \Lambda=0.0098 \mathrm{GeV}^{-2}$ and d) $M_{q}=300 \mathrm{MeV}, \Lambda=0.639 \mathrm{GeV}^{-2}$.

In Fig. 3 the different shapes of the pion DA are shown as they are calculated within the instanton and chiral models for the values of the dynamical quark mass $M_{q}=300 \mathrm{MeV}$ and $M_{q}=$ $125 \mathrm{MeV}$. The parameter $\Lambda$ is defined to fit the pion decay constant in chiral limit $f_{\pi}=85 \mathrm{MeV}$. For smaller $M_{q}$ the pion DA is close to a flat shape. For larger $M_{q}$ it is more sensitive to the nonlocal part of the photon vertex and, in case of the instanton model, it is strongly suppressed in the vicinity of endpoints.

\section{The BABAR data within the instanton and chiral models}

Let us consider the model predictions for the pion transition form factor in the asymmetric kinematics $\left(q_{1}^{2}=Q^{2}, q_{2}^{2}=0\right)$ in the region, where experimental data exist. In Fig. 1, we show the predictions for different values of $M_{q}$. For a quark mass $M_{q}=300 \mathrm{MeV}$ the model dependence is very strong and the theoretical curves are very far from the experimental points. The chiral model overshoots the data, while the instanton model, in correspondence with the standard factorization scenario, shows the asymptotic $1 / Q^{2}$ behavior very early, already at $Q^{2} \sim 1 \mathrm{GeV}^{2}$. It is clearly seen, that in order to describe the BABAR data, one has to take the dynamical quark mass $M_{q} \approx 125$ 
$\mathrm{MeV}$. Then both models have an qualitatively good description, with some preference to the chiral model.

The parameter space that describes the data up to $40 \mathrm{GeV}^{2}$ is rather narrow. For the chiral model it is $M_{q} \approx 125 \pm 10 \mathrm{MeV}$, and for the instanton model it is $M_{q} \approx 130 \pm 5 \mathrm{MeV}$. Thus in this region the instanton model simulate the logarithmically enhanced behavior due to rather flat pion DA. However, the further behavior of the form factor is rather different for different models. The instanton model finally reach its actual asymptotic $1 / Q^{2}$ with the asymptotic coefficient given by

$$
J^{I}=\frac{N_{c}}{4 \pi^{2} f_{\mathrm{PS}, \pi}^{2}} M_{q} \int_{0}^{\infty} d u \frac{u f(u)}{D(u)} \int_{0}^{1} d y \frac{f(y u) m(y u)}{D(y u)} .
$$

For the chiral model the logarithmic growth continues for all $Q^{2}$ with the asymptotics as $Q^{2} \rightarrow \infty$ following from (3.15)

$$
\begin{aligned}
& F_{\pi \gamma^{*} \gamma}^{A s, \chi}\left(0 ; Q^{2}, 0\right)=\frac{1}{Q^{2}} \frac{N_{c}}{12 \pi^{2} f_{\pi}}\left[\int_{0}^{\infty} d u \frac{m^{2}(u)}{D(u)} \ln \left(\frac{Q^{2}}{u}\right)+A^{\chi}\right], \\
& A^{\chi}=\int_{0}^{\infty} d u \frac{m(u)}{D(u)} \int_{0}^{1} d y \frac{m(y u)}{D(y u)}[u-2 m(u) m(y u)] .
\end{aligned}
$$

\section{Conclusions}

As it was stressed in Introduction the main problem to explain the BABAR data is the unstopped growth of the data points for $Q^{2} F_{\pi \gamma \gamma^{*}}\left(Q^{2}\right)$ that is inconsistent with the predicted $Q^{2} F_{\pi \gamma \gamma^{*}}\left(Q^{2}\right) \rightarrow$ constant, following from simple asymptotic properties of the massless quark propagator. The key point, to solve this problem, is to consider the properties of the pion vertex function $F\left(k_{1}^{2}, k_{2}^{2}\right)$ which is the analog of the light-cone pion wave function. There are two possibilities for the momentum dependence of the pion vertex function. In the limit, when one quark virtuality, $k_{1}^{2}$, goes to infinity, and the other, $k_{2}^{2}$, remains finite, the vertex function may not necessarily tend to zero. When it goes to zero, the pion DA $\varphi_{\pi}(x)$, which is a functional of the pion vertex function, is zero at the endpoints, $\varphi_{\pi}(0)=\varphi_{\pi}(1)=0$, with either strong or weak suppression in the neighborhood of the endpoints $x=0$ and $x=1$. For the situation of strong suppression, the asymptotic $1 / Q^{2}$ behavior of the pion form factor in asymmetric kinematics $\left(Q_{1}^{2}=Q^{2}, Q_{2}^{2}=0\right)$ is developed very early, in contradiction with the BABAR data. For weak suppression (resembling a flat distribution amplitude of the pion), the asymptotic $1 / Q^{2}$ behavior is developed quite late, and can give a reasonable description of the data in the BABAR region with a $\ln Q^{2} / Q^{2}$ behavior in this region. For the other case of non-vanishing pion vertex function in the above limit, the pion DA $\varphi_{\pi}(x)$ is not zero at the endpoints, and therefore the asymptotic $\ln Q^{2} / Q^{2}$ behavior persists over the whole range, in particular in the BABAR region.

Concluding we may say, that the BABAR data being unique in their accuracy and covering a very wide kinematical range, are consistent with considerations based on nonperturbative QCD dynamics and may indicate specific properties of the pion wave function.

\section{Acknowledgments}

The author acknowledges partial support from the Russian Foundation for Basic Research projects nos. 10-02-08112 and 10-02-00368. 


\section{References}

[1] G. P. Lepage and S. J. Brodsky, Phys. Rev. D22, 2157 (1980).

[2] S. J. Brodsky and G. P. Lepage, Phys. Rev. D24, 1808 (1981).

[3] CELLO, H. J. Behrend et al., Z. Phys. C49, 401 (1991).

[4] CLEO, J. Gronberg et al., Phys. Rev. D57, 33 (1998), hep-ex/9707031.

[5] The BABAR, B. Aubert et al., Phys. Rev. D80, 052002 (2009), 0905.4778.

[6] A. E. Dorokhov, Eur. Phys. J. C42, 309 (2005), hep-ph/0505007.

[7] J. Terning, Phys. Rev. D44, 887 (1991).

[8] R. D. Bowler and M. C. Birse, Nucl. Phys. A582, 655 (1995), hep-ph/9407336.

[9] R. S. Plant and M. C. Birse, Nucl. Phys. A703, 717 (2002), hep-ph/0007340.

[10] A. E. Dorokhov, JETP Lett. 77, 63 (2003), hep-ph/0212156.

[11] H. Pagels and S. Stokar, Phys. Rev. D20, 2947 (1979).

[12] A. E. Dorokhov, arXiv:1003.4693 [hep-ph].

[13] G. V. Efimov and M. A. Ivanov, Int. J. Mod. Phys. A4, 2031 (1989).

[14] G. V. Efimov and M. A. Ivanov, The Quark Confinement Model of Hadrons (, 1993), Bristol, UK: IOP, $177 \mathrm{p}$.

[15] A. E. Radzhabov and M. K. Volkov, Eur. Phys. J. A19, 139 (2004), hep-ph/0305272.

[16] A. V. Radyushkin, Phys. Rev. D80, 094009 (2009), 0906.0323.

[17] A. E. Dorokhov, Phys. Part. Nucl. Lett. 7, 229 (2010), 0905.4577.

[18] A. E. Dorokhov, Nucl. Phys. Proc. Suppl. 198, 190 (2010), 0909.5111.

[19] D. Diakonov and V. Y. Petrov, Nucl. Phys. B272, 457 (1986).

[20] B. Holdom, J. Terning, and K. Verbeek, Phys. Lett. B245, 612 (1990). 